\title{
Silent Sinus Syndrome in a Female Child with Enophthalmos and Diplopia
}

\author{
${ }^{1}$ Satyawati Mohindra, ${ }^{2}$ Sandeep Mohindra, ${ }^{3}$ Ashok K Gupta
}

\begin{abstract}
Opacification of the maxillary sinus was first published by Montgomery in the precomputerized era. The term silent sinus syndrome (SSS) was firstused by Soparkar et al ${ }^{2}$ in 1994. They defined an uncommon disease characterized by enophthalmos secondary to ipsilateral atelectasis of the maxillary sinus, even without nasal symptoms. The pathophysiology of SSS was demonstrated by Eto et al in 1993. In this report, we are presenting a case of silent sinus syndrome in a girl of pediatric age group, an unusual age of presentation.
\end{abstract}

Keywords: Enophthalmos, Silent sinus syndrome, Hypoglobus.

How to cite this article: Mohindra S, Mohindra S, Gupta AK. Silent S inus Syndrome in a Female Child with Enophthalmos and Diplopia. Clin R hinol An Int J 2014;7(1):40-42.

\section{Source of support Nil}

Conflict of interest None

\section{CASE REPORT}

A 12-year-old girl presented to ENT outpatient department with a history of diplopia and unequal size of the eyes for the past 1 to 2 years. There were no ear nose or throat symptoms. Her past history was suggestive of recurrent attacks of upper respiratory tract infections and sinusitis during early childhood. Clinical examination revealed an enophthalmos of $5 \mathrm{~mm}$ on the right side, hypoglobus, restriction in elevation of the eye and diplopia in superior gaze (Fig. 1A).

Computed tomography (CT) scan (coronal view) revealed a smaller right maxillar sinus, with retraction of all the walls (Fig. 1B). A depression in the floor of orbit on the right side. Osteomeatal complex blockade was evident. A xial sections (Fig. 1C) revealed a hypoplastic maxillary sinus on right side and retraction of right orbit as compared to left side. A $n$ increase in the volume of right gl obe can be seen.

\footnotetext{
${ }^{1,2}$ Associate Professor, ${ }^{3}$ P rofessor

${ }^{1,3}$ Department of Otolaryngology, Head and Neck Surgery Postgraduate Institute of Medical Education and Research Chandigarh, India

${ }^{2}$ Department of Neurosurgery, Postgraduate Institute of Medical Education and Research, Chandigarh, India
}

Corresponding Author: Satyawati Mohindra, Associate Professor, Department of Otolaryngology, Head and Neck Surgery, Postgraduate Institute of Medical Education and Research, Chandigarh, India, Phone: 09914209765, e-mail: satyamohindra@gmail.com, sandeepneuro@gmail.com
With a diagnosis of silent sinus syndrome on right side, the patient was taken up for functional endoscopic sinus surgery to maxillary sinus outflow tract blockade and reconstruction of orbital floor. There was mild DNS, the maxillary sinus was slightly lateralized. The osteomeatal complex was blocked with hypertrophied mucosa. The medial wall of the orbit along with the inferomedial angle was unusually prominent. Orbital floor was reconstructed with the help of septal cartilage via a subciliary approach (Fig. 1D). The patient's diplopia was improved immediately but the lower lid retraction remained for around 6 months. Now after a follow-up of 8 months, her eye symptoms have significantly improved.

\section{DISCUSSION}

Opacification of the maxillary sinus was first published by M ontgomery in the precomputerized era. ${ }^{1}$ The term SSS was first used by Soparkar et $\mathrm{al}^{2}$ in 1994. The pathophysiology of silent sinus syndrome (SSS) was demonstrated by Eto et $\mathrm{al}^{3}$ in 1993. They concluded that enophthalmos stems from remodelling of the bony architecture of the sinus, secondary to it's chronic obstructive disease. Complete obstruction induces continued negative pressure within the sinus, which activates the osteoclasts; in turn, these make the sinus walls thinner, leading to their collapse and the decent of the orbital floor with retraction of it's walls. This then produces hypoglobus and enophthal mos.

It's prevalence is unknown. A pproximately, 100 cases have been described in the literature. It typically affects individuals between the third and fifth decade of life, lacking dominance of gender or side. ${ }^{4}$ We are reporting this in a patient in pediatric age group, with more severe orbital presentation which has not been done earlier.

Though the diagnosis is suspected on clinical examination, it is confirmed on radiology. Computed tomography usually shows inward retraction of sinus walls along with volume loss. This results in enlargement of middle meatus and an increased in orbital volume. There is always retracted and usually thinned orbital floor. IIIneret et al reported five patients with SSS, among them, three patients showed inward retraction of all four sinus walls. A ll of them showed increased orbital volume on the same side. ${ }^{5}$ It is crucial to diagnose SSS correctly because different conditions causing 




Figs 1A to D: (A) Clinical picture depicting enophthalmos, lid retraction and narrowed palpebral fissure on the right side, (B) endoscopic view with a wide middle meatus. The unduly prominent inferomedial angle of the right orbit can be appreciated, (C) CT-axial sections showing enophthalmos on the right side and enlargement of the orbit as compared to the left and (D) CT-coronal sections showing a small right maxillary antrum with blocked osteomeatal complex, in drawing of all the walls and depression of orbital floor

spontaneous enophthal mos requires different treatment and carry a different prognosis. Tumor, trauma, congenital facial asymmetry, diffuse facial lipodystrophy, Parry-Romberg syndrome and linear scleroderma are common sources of incorrect diagnosis of SSS. ${ }^{6}$

The treatment is done to restore a normal ventilation of the maxillary sinus and the normal position of the ocular globe. ${ }^{7}$ A procedure called the Caldwelle-L uc was common in the past. Today, the gold standard treatment to restore sinus function and preserving the maxillary structures is functional endoscopic surgery with uncinectomy and antrostomy. ${ }^{8}$ The orbital floor reconstruction is controversial. There are reports of considering it as a second-stage operation, which is commonly done 2 months after the sinus surgery, due to complete or partial resolution of the enophthalmos, with over-correction in the case of immediate orbital floor reconstruction resulting from the resolution of the hypoventilation and restoration of the normal intrasinus pressure. It is also noticeable that in cases with sever enophthalmos, reconstruction of the orbital floor is inevitable and morbidity, patient discomfort and hospitalization can be reduced by a single-stage operation. Due to negative sinus cultures in most cases, by performing primary reconstruction, there would be no risk of infection. ${ }^{9}$ We performed orbital reconstruction primarily to relieve diplopia which is quite incapacitating. Due to displacement of orbital wall and presentation of the inferomedial angle in the meatus it was safely done via subciliary approach. The patient is significantly improved at 8 months of follow-up.

\section{CONCLUSION}

Though silent sinus syndrome presents in later age group, it may begin in padiatric age group as evident from our case. Since, the complications of SSS, such as upper lid retraction, malar depression, facial asynmetry, diplopia and enophthalmos, can be prevented by early diagnosis, a high index of suspicion must be exercized.

\section{REFERENCES}

1. M ontgomery WW. M ucocele of the maxillary sinus causing enophthalmos. Eye Ear Nose Throat M on 1964 M ay;43:41-44.

2. Soparkar CN, Patrinely JR, Cuaycong M J, Dailey RA, K ersten RC, Rubin PA, Linberg J V, H oward GR, D onovan DT, M atoba $A Y$, et al. The silent sinus syndrome: a cause of spontaneous enophthalmos. Ophthalmology 1994 A pr;101(4):772-778. 
3. Eto RT1, House JM. Enophthalmos, a sequela of maxillary sinusitis. A m J Neuroradiol 1995 A pr;16(Suppl 4):939-941.

4. CardesínA, EscamillaY, R omera M, M olina A A. Single surgical step for endoscopic surgery and orbital reconstruction of a silent sinus syndrome. A cta Otorhinolaryngol Esp 2013 JulA ug;64(4):297-299.

5. Sheikhi M 1, Jalalian F. The silent sinus syndrome. Dent Res J (I sfahan) $2013 \mathrm{M} \mathrm{ar} ; 10(2): 264-267$.

6. Monos $T$, Levy J, Lifshitz $T$, Puterman $M$. The silent sinus syndrome. Isr M ed A ssoc J 2005 M ay;7(5):333-335.
7. Sánchez-Dalmau B F, Pascual L, L ao X, M aiz J. Sinus syndrome, an uncommon cause of enophthalmos. A rch Soc Esp O ftalmol 2008;83:125-128.

8. Sesenna E, Oretti G, Anghinoni ML, Ferri A. Simultaneous management of the enophthal mos and sinus pathology in silent sinus syndrome: A report of three cases. J Craniomaxillofac Surg 2010;38:469-472.

9. Behbehani R, Vacareza N, Bilyk JR, Rubin PA, Pribitkin EA. Simultaneous endoscopic antrostomy and orbital reconstruction in silent sinus syndrome. Orbit 2006;25:97-101. 